\title{
Network Lifetime Analysis in IOT Environment in Healthcare Sectors Using Deep Learning Routing Approach
}

\author{
Janaki $\mathrm{K}^{\mathrm{a}, 1}$ \\ a Assistant Professor in Computer Science, Bharathi Women's College (Autonomous), \\ Chennai
}

\begin{abstract}
The Internet of Things (IoT) provides an improved flexibility in data collection, network deployment and data transmission to the sink nodes. However, depending on the application, the IoT network tends to consume lot of power from the individual devices. Various conventional solutions are provided to reduce the consumption of energy but most methods focus on increasing the data acquisition speed, data transmission and routing capabilities. However, these methods tend to fall under the trade-off between these three factors. Hence, in order to maintain the trade-off between these constraints, a viable solution is developed in this paper. A deep learning-based routing is built considering the faster acquisition of data, faster data transmission and routing path estimation with increasing path estimation. The paper models a Deep belief Network (DBN) to route the data considering all these constraints. The experimental validation is conducted to check the network lifetime, energy consumption of IoT nodes. The results show that the DBN offers greater source of flexibility with increased data routing capabilities than other methods.
\end{abstract}

Keywords. Multi-layered perceptron, routing, energy consumption, IOT.

\section{Introduction}

The IoT is a new technology that aims to take care of physical objects in our everyday lives. The IoT core concept is fitted with interconnected sensors that make it into a smarter entity and devices that are clearly linked and transmitted to the Internet. IoT is designed to work together between the machines, leading to complex tasks. Consequently, these instruments have the capacity to store, process and transfer data. Only existing technologies including smart sensor networks, Radio Frequency Identification (RFID), Near-Field-Communication (NFC), cell technology, and the internet make convergence possible. Through incorporating this technology into a single device, the IoT architecture is developed from a variety of processors, from strong and complicated servers, into the RFID Tag.In particular, some devices are supposed to have low efficiency and performance considering their heterogeneous IoT environment. Because of inadequate

\footnotetext{
${ }^{1}$ Janaki K, Department of Computer Science, Bharathi Women's College (Autonomous), Chennai. E-mail: janakikothandan1972@gmail.com.
} 
processing, limited capacity, low energy, and low latency, network machines have low throughput and a high latency [1].

Traditional solutions to the routing dilemma of such networks do not function appropriately when taken into account limitations such as the need for a significant volume of money and energy.In recent years, not much research has been carried out to improve the minimal conventional solutions. The problems and the routing algorithm ought to be changed to use IoT in common citizens. Lack of robust data forwarding is a major impediment to data transfer between source and destination in vast networks. The Internet of Things poses unique problems and limitations that were not taken into consideration when designing past routing protocols, each with different network requirements. In this situation, it is essential to handle rapidly evolving network topologies. As we have proposed in this paper, an IoT routing protocol is to choose the best path.Intelligent agents choose routes based on battery capacity, plunging size, usable bandwidth, and path stability rating based on their proposed system.

The intelligent way to prepare nodes for optimal paths is by using the Deep belief Network (DBN). It maintains trade-off between these energy efficiency and power consumption. A DBN is built considering the faster acquisition of data, faster data transmission and routing path estimation with increasing path estimation. The paper models a DBN to route the data considering all these constraints. The experimental validation is conducted to check the network lifetime, energy consumption of IoT nodes. The results show that the DBN offers greater source of flexibility with increased data routing capabilities than other methods.

\section{Related works}

Devices that are linked to different computing capacities were enhanced through the development of IoT. Multimedia streaming on IoT cannot adapt to heterogeneous devices with a high level of service and, perhaps, it is impossible for a system to accept data from all devices. Heterogeneity of devices in order to react to requirements. The authors of [2] proposed an algorithm for balancing the layers in the following problem. For IoT multimedia streams, this algorithm uses network decoding, whereas for multimedia network entity streaming, it uses network coding. The writers used primitive and primal decomposition in this article. The Lyapunov principle was often used to show that the suggested algorithm converges and maintains its stability. The usage of wireless sensor networks is highly prevalent in linking the real environment with the IoT knowledge world. Around the same time, the WSN has a greater need for frequent communication with the power supply. In the case of a multicast transmission, the base station transmits to a single recipient (which is called a community in this context), whereas multiple beneficiaries get the info. To answer this question, we need to break it down into the Steiner tree dilemma, which has been shown to be an NP-complete problem.

An example of a modern multicast routing protocol is the multicast routing algorithm used in [3], which solves the issue of service consistency constraints. Link estimates are unintended because they are being aggregated or because the load of traffic changes suddenly. Furthermore, since the relation requirements are not aggregate, the deterministic algorithms of the multi-thread tree do not apply. Random parameters, on the other side, take relations and offer them distinct, concrete descriptions, which can 
be provided as inputs for quantitative modelling or qualitative characterization. The developers of [4] looked at how to use information-centered networks to make it easier to deploy Internet artefacts and have a modular framework that allows IoT to operate better in such a network. The naming requirements, corporate procedures, protection, and energy conservation were all taken into account in the designed architecture. The writers have reviewed the total amount of physical interaction that is made through IoT device protection mechanisms in this article.

In the text, the authors of [5] came up with a greedy method with Small World Model Properties for IoT sensor networks (SWMP). The GMSW identifies the two greedy nodes as separate nodes in the network and uses them as a criteria for assigning significance to numerous other nodes. They implemented the concept of node local value, which they defined using these various parameters. They implement an algorithm which enables them to modify the network in order to manipulate the characteristics of a narrow global model, which is done by using shortcuts between individual nodes that are focused on the location of those nodes.

The authors of [6] recommended a content-conscious approach for sensor nodes to shape SSONs in the form of Ant clusters in knowledge awareness, which implements a similar strategy to SSONs in form of Ant clusters inside a cluster of linked sensor nodes. After classifying the sensors into SSONs based on their structure, the next step is to conduct the analysis. After the nodes are clustered using the suggested antclust algorithm, the nodes are joined to the network. Another beneficial aspect of these new procedures is that they decrease expenses while also keeping the network operational.

The presented solution, according to [7], is built on a novel real-time IoT routing algorithm, where generalised information decisions are utilised to increase the overall security and response time of IoT transmissions. In addition, the authors have developed a delay iterative method (DIM) to address the issue of missing valid routes, which is entirely based on delay calculations. in particular In addition, the REPC, a current transmission device, collects and reveals additional information about the energy level in the nodes, which makes for a more efficient distribution of network resources. This research done by [8] authors compared routing protocols and IoT Medium Access Control (MAC), and their conclusions indicated that existing protocols do not provide for cooperation between routing strata and MAC layers; new requirements were therefore proposed as a consequence of this discovery. These requirements enable routing protocols to perform necessary modifications, such as to the MA and routing protocols, while also allowing adjustment of the parameters as needed. The authorization standards in 9 included consistency of Internet connectivity specifications and methodologies for enhancing service efficiency parameters. Any model can be understood in the context of a computing analogy, and the dynamic system model of service efficiency is simplified. The algorithm has been modernised by integrating quality service technology with complicated service technology.

In this paper [9], the authors addressed the issue of misplaced wearable IoT devices by using their MAC address to track its location, this can be done through examining the access points in which the devices are currently connected. In this work [10], proposed a recommendation system for crop yield prediction using Support Vector Machine and Decision Tree algorithm. This system is IoT based, which automatically determines the suitable crop by analysing the characteristics of the soil and climatic conditions and recommends the same to optimize the yield. 
In [11], they introduced a secure energy efficient protocol for efficient routing in Wireless Sensor Networks. This protocol works based on the sender responsive technique which uses energy for node mobility prediction and when connection failure occurs. It implements fuzzy rules and consider trust value of a node for secure routing and to improve the packet delivery ratio.

\section{Proposed Method}

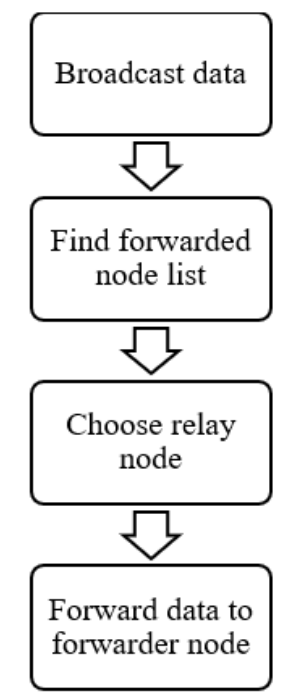

Figure 1. Architecture of IoT assisted DBN Routing

As literature indicates that an IoT energy consumption has a significant effect on the existence and efficiency, it is therefore essential that energy efficient, protocols for routing be designed to increase overall network life and improve the quality of the IoT network. In this paper, we use DBN for discovery of routes and maintenance of routes, where the details are given below.

Step 1: Use DBN for broadcasting the packet from source node to destination nodes by finding the forwarder node list.

Step 2: Choose relay among the IoT sensor nodes from the forwarder list.

Step 3: Forward the collected IoT data to the forwarder node or relay node.

\subsection{Deep Belief Network}

Consider a set $(\mathrm{v}, \mathrm{h})$ with the validation function $\mathrm{E}(\mathrm{v}, \mathrm{h} \mid \theta)$ that acts as an energy function for training the deep belief network and it is expressed as below:

$$
E(v, h \mid \theta)=-\sum_{i=1}^{n} a_{i} v_{i}-\sum_{j=1}^{m} b_{j} h_{j}-\sum_{i=1}^{n} \sum_{j=1}^{n} v_{i} w_{i j} h_{j}
$$


where in Eq. (1), $\theta$ - DBN parameter $\theta=\left\{W_{i j}, a_{i}, b_{j}\right\}$ with $\mathrm{w}$ is considered as the connection weight $\mathrm{a}$ is considered as the visible hidden layer bias and $\mathrm{b}$ is considered as the hidden layer bias.

The joint probability distribution for an output and hidden layer (h) is hence expressed as follows:

$$
\begin{gathered}
P(v, h \mid \theta)=\frac{e^{-E(v, h \mid \theta)}}{Z(\theta)} \\
Z(\theta)=\sum_{v, h} e^{-E(v, h \mid \theta)}
\end{gathered}
$$

$\operatorname{CDF}(a, b)$ or the conditional distribution function is thus expressed in the form of Gibbs sampling process and it is expressed as below:

$$
\begin{aligned}
& P\left(h_{j}=1 \mid v, \theta\right)=\text { sigmoid }\left[b_{j}+\sum_{i} v_{i} w_{i j}\right] \\
& P\left(v_{j}=1 \mid v, \theta\right)=\text { sigmoid }\left[a_{j}+\sum_{i} h_{i} w_{j i}\right]
\end{aligned}
$$

With the expression in Eq. (3), the probability of hidden layer can thus be derived for active state. DBN tends to provide symmetric feature with the probability over activation state, which is regarded as visible states and hidden layer states $h$.

\subsection{Route discovery phase}

In the case of an IoT network, a node graph could be used. When two devices have the ability to communicate directly with each other through their radio system, we name them connected.Since one of these two nodes would have more powerful transmitters than the other, there is a chance that contact will form between the nodes but not the other way around. Furthermore, when two nodes are in the radio spectrum, there is no guarantee that they can communicate; buildings, hills, or other obstacles will prevent them from communicating. The features (identifier number, application ID) in a local table comprising packet records are searched first, and if the packet has already been retrieved and analysed, the application ID is used instead. The package will be discarded if it has been duplicated, and its sorting will be completed. If the packet attribute is not duplicated, it will be imported into the record table, which means that it will not be stored in the future, which therefore will preclude the loop from routing.

\subsection{Route Maintained Phase}

As the IoT nodes may be switched off in this form of network to minimize the level of the battery, the network configuration is often modified. This protocol is supposed to solve this problem somehow. The Hello broadcast containing the identity and location 
information, is also transmitted alternatively to each node on the networkThe editor of the communication will be alerted if no answer is received. In this instance, the editor will know that his neighbour is unable to respond, and that the link has been severed. It is expected that the neighbours react to the post.

\section{Results and Discussions}

This section presents the results of various metrics by the proposed method that includes energy consumption, network throughput, network lifetime and network delay in delivering the packets to the destination with various existing techniques like MLP (Multi-Layer Perceptron), RNN (Recurrent Neural Network), DBN (Deep Belief Networks). The Figure 2 shows the results of energy consumption between the sensor nodes. It is evident that the DBN maintains the task of route allocation and route maintenance effectively such that the data packets are transmitted effectively from source node to destination node. The results of simulation shows that the proposed method attains higher rate of energy conservation than other existing models. The effects of network throughput between the

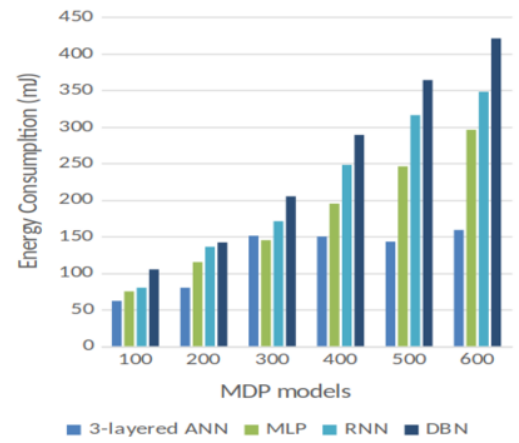

Figure 2. Energy Consumption

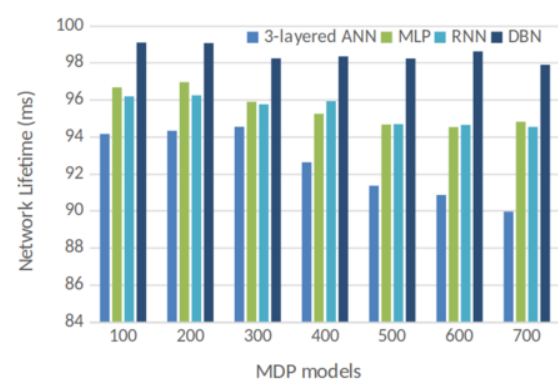

Figure 4. Network Lifetime

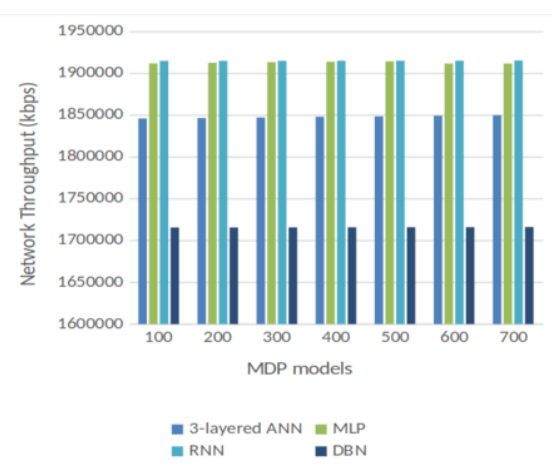

Figure 3. Network Throughput

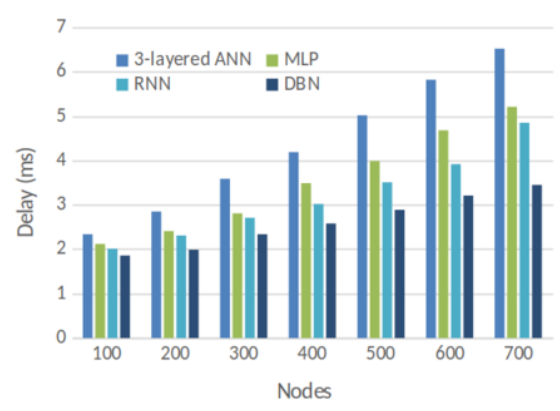

Figure 5. Network Delay

sensor nodes are seen in Figure 3. It is clear that the DBN improves network throughput by allocating routes optimally and avoiding communication failures, ensuring that data packets are easily transferred from source to destination nodes. Simulation findings 
reveal that the proposed approach achieves a higher network throughput rate than other current models. The Figure 4 shows the results of network lifetime between the sensor nodes. It is evident that the DBN increases the network lifetime using optimal allocation of routes without transmission failure so that the data packets are transmitted effectively from source node to destination node. The results of simulation shows that the proposed method attains higher rate of network lifetime than other existing models. The Figure 5 shows the results of end-to-end delay between the sensor nodes. It is evident that the DBN reduces the delay including transmission delay, reception delay and propagation delay in delivery the packets from source node to destination node. The results of simulation shows that the proposed method attains reduced rate of delay than other existing models.

\section{Conclusion and Future Enhancement}

A DBN provides faster routing of data, faster data transmission and routing path estimation with increasing path estimation. The DBN routes the data considering all these constraints that includes path estimation, data transmission and routing parameters. The validation shows that the DBN attains increased network lifetime, reduced energy consumption of IoT nodes. The results show that the DBN offers greater source of flexibility with increased data routing capabilities than other methods. Data encryption will be allowed in the future when data is being transferred between source and destination nodes. Furthermore, the usage of blockchain technologies will allow for the most efficient and reliable data transfer from the source to the destination nodes.

\section{References}

[1] Duraipandian M. Performance evaluation of routing algorithm for Manet based on the machine learning techniques. Journal of trends in Computer Science and Smart technology (TCSST). 2019 Sep;1(01):2538.

[2] Troia S, Rodriguez A, Martín I, Hernández JA, De Dios OG, Alvizu R, Musumeci F, Maier G. Machinelearning-assisted routing in SDN-based optical networks. In 2018 European Conference on Optical Communication (ECOC) 2018 Sep 23 (pp. 1-3). IEEE.

[3] Tang Y, Cheng N, Wu W, Wang M, Dai Y, Shen X. Delay-minimization routing for heterogeneous VANETs with machine learning based mobility prediction. IEEE Transactions on Vehicular Technology. 2019 Feb 15;68(4):3967-79.

[4] Yuvaraj N, Kousik NV, Jayasri S, Daniel A, Rajakumar P. A survey on various load balancing algorithm to improve the task scheduling in cloud computing environment. J Adv Res Dyn Control Syst. 2019;11(08):2397-406.

[5] Yao H, Yuan X, Zhang P, Wang J, Jiang C, Guizani M. Machine learning aided load balance routing scheme considering queue utilization. IEEE Transactions on Vehicular Technology. 2019 Jun 10;68(8):7987-99.

[6] Yuvaraj N, Raja RA, Karthikeyan T, Kousik NV. Improved Privacy Preservation Framework for CloudBased Internet of Things. In Internet of Things 2020 Dec 29 (pp. 165-174). CRC Press.

[7] Tabrizi AF, Rakai L, Darav NK, Bustany I, Behjat L, Xu S, Kennings A. A machine learning framework to identify detailed routing short violations from a placed netlist. In2018 55th ACM/ESDA/IEEE Design Automation Conference (DAC) 2018 Jun 24 (pp. 1-6). IEEE.

[8] Kannan S, Dhiman G, Natarajan Y, Sharma A, Mohanty SN, Soni M, Easwaran U, Ghorbani H, Asheralieva A, Gheisari M. Ubiquitous vehicular ad-hoc network computing using deep neural network with iot-based bat agents for traffic management. Electronics. 2021 Jan;10(785):1-16. 
[9] Sai Ramesh L, Sundar SS, Selvakumar K, Sabena S. Tracking of Wearable IoT Devices Through WAP Using Intelligent Rule-Based Location Aware Approach. Journal of Information \& Knowledge Management. 2021 Feb 29;20(supp01):2140005.

[10] Reshma R, Sathiyavathi V, Sindhu T, Selvakumar K, SaiRamesh L. IoT based Classification Techniques for Soil Content Analysis and Crop Yield Prediction. In 2020 Fourth International Conference on ISMAC (IoT in Social, Mobile, Analytics and Cloud)(I-SMAC) 2020 Oct 7 (pp. 156-160). IEEE.

[11] Dhanalakshmi B, SaiRamesh L, Selvakumar K. Intelligent energy-aware and secured QoS routing protocol with dynamic mobility estimation for wireless sensor networks. Wireless Networks. 2021 Feb;27(2):1503-14.

[12] Anand J, Perinbam J, Meganathan D. Design of GA-based Routing in Biomedical Wireless Sensor Networks. International Journal of Applied Engineering Research (IJAER). 2015;10(4):9281-92.

[13] Sangeetha SB, Blessing NW, Yuvaraj N, Sneha JA. Improving the training pattern in back-propagation neural networks using holt-winters' seasonal method and gradient boosting model. In Applications of Machine Learning 2020 (pp. 189-198). Springer, Singapore.

[14] Anand J, Jeevaratinam RP, Deivasigamani M. Performance of Optimized Routing in Biomedical Wireless Sensor Networks Using Evolutionary Algorithms. Comptes rendus de l'Académie bulgare des Sciences. 2015 Aug 1;68(8):1049-55.

[15] Dudukovich R, Hylton A, Papachristou C. A machine learning concept for DTN routing. In 2017 IEEE International Conference on Wireless for Space and Extreme Environments (WiSEE) 2017 Oct 10 (pp. 110-115). IEEE.

[16] Luong NT, Vo TT, Hoang D. FAPRP: A machine learning approach to flooding attacks prevention routing protocol in mobile ad hoc networks. Wireless Communications and Mobile Computing. 2019 Jan 1;2019(6869307):1-17.

[17] Rupasinghe T, Cooray P. Machine Learning-Based Parameter Tuned Genetic Algorithm for Energy Minimizing Vehicle Routing Problem. 2017;2017(3019523):1-13.

[18] Yu C, Lan J, Guo Z, Hu Y. DROM: Optimizing the routing in software-defined networks with deep reinforcement learning. IEEE Access. 2018 Oct 23;6:64533-9.

[19] Militani DR, de Moraes HP, Rosa RL, Wuttisittikulkij L, Ramírez MA, Rodríguez DZ. Enhanced Routing Algorithm Based on Reinforcement Machine Learning-A Case of VoIP Service. Sensors. 2021 Jan;21(504):1-32.

[20] Arappali N, Rajendran GB. MANET security routing protocols based on a machine learning technique (Raspberry PIs). Journal of Ambient Intelligence and Humanized Computing. 2021 Jun;12(6):6317-31.

[21] Anand J, Perinbam JR, Meganathan D. Q-learning-based optimized routing in biomedical wireless sensor networks. IETE Journal of Research. 2017 Jan 2;63(1):89-97.

[22] Zeng W, Zhang B, Davoodi A. Analysis of security of split manufacturing using machine learning. IEEE Transactions on Very Large Scale Integration (VLSI) Systems. 2019 Aug 6;27(12):2767-80.

[23] Proietti R, Chen X, Zhang K, Liu G, Shamsabardeh M, Castro A, Velasco L, Zhu Z, Yoo SB. Experimental demonstration of machine-learning-aided QoT estimation in multi-domain elastic optical networks with alien wavelengths. Journal of Optical Communications and Networking. 2019 Jan 1;11(1):A1-10.

[24] Sharma DK, Dhurandher SK, Agarwal D, Arora K. kROp: k-Means clustering based routing protocol for opportunistic networks. Journal of Ambient Intelligence and Humanized Computing. 2019 Apr;10(4):1289-306.

[25] Tang F, Mao B, Fadlullah ZM, Kato N, Akashi O, Inoue T, Mizutani K. On removing routing protocol from future wireless networks: A real-time deep learning approach for intelligent traffic control. IEEE Wireless Communications. 2017 Oct 27;25(1):154-60.

[26] Caglayan MU. G-networks and their applications to machine learning, energy packet networks and routing: Introduction to the special issue. Probability in the Engineering and Informational Sciences. 2017 Oct;31(4):381-95.

[27] Troia S, Martinez DE, Martín I, Zorello LM, Maier G, Hernández JA, de Dios OG, Garrich M, RomeroGázquez JL, Moreno-Muro FJ, Mariño PP. Machine learning-assisted planning and provisioning for SDN/NFV-enabled metropolitan networks. In 2019 European Conference on Networks and Communications (EuCNC) 2019 Jun 18 (pp. 438-442). IEEE.

[28] Etengu R, Tan SC, Kwang LC, Abbou FM, Chuah TC. AI-Assisted Framework for Green-Routing and Load Balancing in Hybrid Software-Defined Networking: Proposal, Challenges and Future Perspective. IEEE Access. 2020 Sep 14;8:166384-441. 